\title{
ON A THEOREM OF MINKOWSKI'S
}

A. C. WOODS

Let $K$ be a closed strictly convex body in $R_{n}$ symmetric in the origin 0 . Let $\Lambda$ be a $K$-admissible lattice, i.e. apart from 0 no point of $\Lambda$ is in the interior of $K$. A theorem of Minkowski's (1) is that there are at most $2^{n}-1$ pairs of points $\pm X$ of $\Lambda$ that lie on the boundary of $K$. I note here that this is a special case of a theorem which applies to any lattice.

Let $K$ be as above and let $\Lambda$ be an arbitrary lattice in $R_{n}$, so not necessarily $K$-admissible. The $n$ numbers $\mu_{1}(\Lambda), \mu_{2}(\Lambda), \cdots, \mu_{n}(\Lambda)$, called the successive minima of $\Lambda$ with respect to $K$, are defined as the least upper bounds respectively of numbers $c_{1}, c_{2}, \cdots, c_{n}$ with the property that $c_{i} K$ contains at most $i-1$ linearly independent points of $\Lambda$ within its interior. Let $X_{1}, X_{2}, \cdots, X_{n}$ be $n$ linearly independent points of $\Lambda$ such that $\mu_{i}(\Lambda) K$ contains $X_{1}, X_{2}, \cdots, X_{i}$ for $i=1,2, \cdots, n$. Let $Z_{1}, Z_{2}, \cdots, Z_{n}$ be a basis of $\Lambda$ such that

$$
X_{i}=\sum_{j=1}^{i} g_{i j} Z_{j} \quad(i=1,2, \cdots, n)
$$

where the coefficients $g_{i j}$ are integers with $g_{i i}>0$. Denote by $X_{i 1}$, $X_{i 2}, \cdots, X_{i r_{i}}$ all the points of $\Lambda \bigcap \mu_{i}(\Lambda) K$ of the form

$$
X_{i s}=\sum_{j=1}^{i} g_{i j}^{(s)} Z_{j}
$$

where the coefficients $g_{1 j}^{(s)}$ are integers with $g_{i 1}^{(s)}>0$. Then we have the result

THEOREM. $\sum_{i=1}^{n} r_{i} \leqq 2^{n}-1$.

Proof. Take coordinates such that $Z_{1}, Z_{2}, \cdots, Z_{n}$ are the points $(1,0,0, \cdots, 0),(0,1,0, \cdots, 0), \cdots,(0,0, \cdots, 0,1)$ respectively. Then $\Lambda$ is the set of points with integral coordinates. We say that two points of $\Lambda$ are congruent modulo 2 if the corresponding differences between their coordinates are divisible by 2 . From the definition of the successive minima of $\Lambda$ it follows that every one of the points $X_{i s}$ is primitive and therefore in particular no one of them is congruent to $0=(0,0, \cdots, 0)$ modulo 2 . We assert that no two of these points can be congruent modulo 2. For assume that this assertion is false. Then for two distinct pairs of indices $i, s$ and $i^{\prime}, s^{\prime}$

Received by the editors November 25, 1957. 
the points $X_{i s}$ and $X_{i^{\prime} s^{\prime}}$ are congruent modulo 2 . Hence $\left(X_{i s}+X_{i^{\prime} s^{\prime}}\right) / 2$ and $\left(X_{i s}-X_{i^{\prime} s^{\prime}}\right) / 2$ are points of $\Lambda$. If $\left(X_{i s}+X_{i^{\prime} s^{\prime}}\right) / 2=X_{i s}$ then $X_{i s}=X_{i^{\prime} s^{\prime}}$ which is impossible since by definition distinct pairs of suffixes yield distinct points, therefore $\left(X_{i s}+X_{i^{\prime} s^{\prime}}\right) / 2 \neq X_{i s}$. Similarly $\left(X_{i s}+X_{i^{\prime} s^{\prime}}\right) / 2 \neq X_{i^{\prime} s^{\prime}}$. If $\left(X_{i s}-X_{i^{\prime} s^{\prime}}\right) / 2=X_{i s}$ then $X_{i s}=-X_{i^{\prime} s^{\prime}}$ and therefore in particular $i=i^{\prime}$. Hence $g_{i i}^{(s)}=-g_{i^{\prime} i^{\prime}}^{\left(s^{\prime}\right)}$, but as $g_{i i}^{(s)}$ and $g_{i i}^{\left(s^{\prime}\right)}$ are positive integers this is impossible, hence $\left(X_{i s}-X_{i^{\prime} s^{\prime}}\right) / 2 \neq X_{i 8}$. Similarly $\left(X_{i s}-X_{i^{\prime} s^{\prime}}\right) / 2 \not \neq-X_{i^{\prime} s^{\prime}}$. There is no loss of generality in assuming that $i \geqq i^{\prime}$. It follows that $X_{i s}$ and $X_{i^{\prime} s^{\prime}}$ are contained in $\mu_{i}(\Lambda) K$. From the strict convexity of $K$ it follows that $\left(X_{i s}+X_{i^{\prime} s^{\prime}}\right) / 2$ and $\left(X_{i s}-X_{i^{\prime} s^{\prime}}\right) / 2$ are in the interior of $\mu_{i}(\Lambda) K$. Now

$$
X_{i s}=\left(X_{i s}+X_{i^{\prime} s^{\prime}}\right) / 2+\left(X_{i s}-X_{i^{\prime} s^{\prime}}\right) / 2
$$

so that $X_{i s}$ is linearly dependent on two points of $\Lambda$ which lie in the interior of $\mu_{i}(\Lambda) K$. As this is impossible the assertion is proved. The assertion implies that no two of the points $X_{i s}$ can lie in the same residue class modulo 2 and since as we have already seen no one of these points is congruent to 0 modulo 2 it follows that there are at most $2^{n}-1$ such points and the theorem is proved.

Minkowski extended his result to convex bodies which are not strictly convex by showing that if $\Lambda$ is an admissible lattice of an arbitrary convex body $K$ symmetric in the origin then there are at most $3^{n}-1$ points of $\Lambda$ on the boundary of $K$. Here the extension breaks down for let $K$ be a convex body symmetric in the origin such that the boundary of $K$ contains a line segment. Take coordinates so that one such line segment has the endpoints $(1,1,0, \cdots, 0)$, $(-1,1,0, \cdots, 0)$. For an arbitrary pair of positive integers $M, N$ denote by $\Lambda(M, N)$ the lattice generated by the point $\left(N^{-1}, 0,0, \cdots, 0\right),(0,1,0, \cdots, 0),(0,0, M, 0, \cdots, 0), \cdots$, $(0,0, \cdots, 0, M)$. As $K$ is bounded so for all sufficiently large values of $M, r_{1}=1$ and $r_{2} \geqq 2 N+1$ whence $\sum_{i+1}^{n} r_{i} \geqq 2 N+2$ which tends to infinity with $N$.

\section{REFERENCE}

1. H. Minkowski, Geometrie der Zahlen, Leipzig, 1928.

Tulane UNIVERsity of Louisiana 